Urologe 2016 55 (Suppl 1):S2-S4 DOI 10.1007/s00120-016-0212-4

Online publiziert: 18. August 2016

๑) Springer-Verlag Berlin Heidelberg 2016

CrossMark

Jonas Busch

Klinik für Urologie, Charité Universitätsmedizin Berlin, Berlin, Deutschland

\title{
Auswahlverfahren wissenschaftlicher Beiträge zum DGU-Kongress 2016
}

Für den 68. Kongress der Deutschen Gesellschaft für Urologie e. V. (DGU), der 2016 erneut in Leipzig stattfindet, wurden 648 Abstracts über 46 Themengebiete eingereicht. Die Einreichung erfolgte, wie bereits in den vergangenen Jahren, über das Online-Portal der Firma Pharma Service (Hannover).

Alle Beiträge konnten sowohl in deutscher als auch in englischer Sprache eingereicht werden. Zudem durften international bereits vorgestellte Arbeiten nochmals eingereicht werden. Insgesamt war die Anzahl an eingereichten Arbeiten auch in diesem Jahr erneut rückläufig.

Die Abstracts wurden, nach Themengebieten sortiert, von jeweils maximal 4 erfahrenen Gutachtern bewertet. Neben einer Benotung nach dem Prinzip von Schulnoten von 1-6 wurde dabei auch eine Vorauswahl potentiell preiswürdige Abstracts vorgenommen. Anschließend wurden die bewerteten Abstracts von den Obergutachtern (welche sich aus den Mitgliedern der Programmkommission zusammensetzten) auf ihre Korrektheit hin überprüft (z. B. Doppelveröffentlichungen, Mehrfacheinreichungen etc.) (- Tab. 1). Eine weitere Aufgabe der Obergutachter bestand darin, die bewerteten Abstracts zu wissenschaftlichen Sitzungen zusammenzufügen.

Im Rahmen der finalen Sitzung der Programmkommission wurden diese bereits vorbereiteten Vortragssitzungen dann vorgestellt und ggf. noch geringfügig modifiziert bzw. Beiträge noch anderen Sitzungen zugeordnet, die von anderen Obergutachtern bearbeitet wurden und sich inhaltlich ergänzten.

Insgesamt belief sich Anzahl der angenommenen Beiträge auf 366 von 648, was eine Quote von 56,48 \% entspricht. Neun angenommene Abstracts wurden nach Annahme von den Autoren zurückgezogen. Die konstant hohe Annahmequote entspricht damit den Werten aus den Vorjahren (2012: 55,8\%, 2013: $56 \%$; 2014: $55 \% ; 2016$ 63,8\%), ist aber auch auf die individuelle Betrachtung der Abstracts zurückzuführen. In mehreren Fällen erfolgte zudem die Bitte der Programmkommission an verschiedene Autoren, mehrere Beiträge zu einem Abstract zusammenzufassen.

Unser Dank gilt allen einreichenden Autoren, den Gutachtern, den Mitgliedern der Programmkommission mit ihren Obergutachtern, Frau Chaya und Frau Fus sowie der gesamten DGUGeschäftsstelle und der Firma Pharma Service.

Die Attraktivität und Nachhaltigkeit eines wissenschaftlichen Kongresses lebt von der Präsentation von Originalarbeiten und deren kollegialer Diskussion. Zudem kann dadurch der Facettenreichtum der nationalen wie internationalen urologischen Forschung dargestellt und belegt werden, dass die Urologie mehr umfasst. Wir hoffen auf ein breit gefächertes, interessantes Programm, lebendige Diskussionen und eine weitere Stärkung der urologischen Forschung.

\section{Korrespondenzadresse}

Priv. Doz. Dr. J. Busch

Klinik für Urologie, Charité Universitätsmedizin Berlin

Charité Platz 1, 10117 Berlin, Deutschland jonas.busch@charite.de

Interessenkonflikt. J. Busch gibt an, dass kein Interessenkonflikt besteht. 
Tab. 1 Wissenschaftliche Beiträge zum DGU-Kongress 2016

\begin{tabular}{|c|c|c|c|c|c|c|c|c|c|}
\hline & Themen & $\begin{array}{l}\text { Anzahl } \\
\text { einge- } \\
\text { reichter } \\
\text { Beiträge }\end{array}$ & Video & Oral & $\begin{array}{l}\text { Ange- } \\
\text { nommen }\end{array}$ & $\begin{array}{l}\text { Abge- } \\
\text { lehnt }\end{array}$ & $\begin{array}{l}\text { Zurückgezo- } \\
\text { gene/zusam- } \\
\text { mengefasste } \\
\text { Beiträge }\end{array}$ & Quote & $\begin{array}{l}\text { Durchschnitts- } \\
\text { note }\end{array}$ \\
\hline 2 & BPH/Unterer Harntrakt - Experimentell & 9 & 0 & 7 & 7 & 2 & 0 & 77,78 & 1,08 \\
\hline 4 & BPH/Unterer Harntrakt - Therapie & 29 & 1 & 10 & 11 & 18 & 0 & 37,93 & 1,70 \\
\hline 6 & $\begin{array}{l}\text { Inkontinenz/Neurourologie - Experi- } \\
\text { mentell }\end{array}$ & 6 & 0 & 5 & 5 & 1 & 0 & 83,33 & 0,77 \\
\hline 7 & $\begin{array}{l}\text { Inkontinenz/Neurourologie - Diagnos- } \\
\text { tik }\end{array}$ & 6 & 0 & 4 & 4 & 2 & 0 & 66,67 & 0,62 \\
\hline 8 & Inkontinenz/Neurourologie - Therapie & 25 & 0 & 10 & 10 & 13 & 2 & 40,00 & 1,54 \\
\hline 10 & $\begin{array}{l}\text { Niere, Oberer Harntrakt - Physiologie, } \\
\text { Pathophysiologie }\end{array}$ & 3 & 0 & 0 & 0 & 3 & 0 & 0,00 & 0,00 \\
\hline 11 & $\begin{array}{l}\text { Niere, Oberer Harntrakt - Transplantati- } \\
\text { on }\end{array}$ & 9 & 0 & 9 & 9 & 0 & 0 & 100,00 & 1,39 \\
\hline 13 & $\begin{array}{l}\text { Nieren- und Nebennierentumoren - } \\
\text { Experimentell }\end{array}$ & 20 & 0 & 9 & 9 & 11 & 0 & 45,00 & 1,39 \\
\hline 14 & $\begin{array}{l}\text { Nieren- und Nebennierentumoren - } \\
\text { Diagnostik }\end{array}$ & 1 & 0 & 1 & 1 & 0 & 0 & 100,00 & 0,15 \\
\hline 15 & $\begin{array}{l}\text { Nieren- und Nebennierentumoren - } \\
\text { Prognose und Prädiktion }\end{array}$ & 21 & 0 & 9 & 9 & 11 & 1 & 42,86 & 1,39 \\
\hline 16 & $\begin{array}{l}\text { Nieren- und Nebennierentumoren - } \\
\text { Operative Therapie }\end{array}$ & 22 & 1 & 8 & 9 & 13 & 0 & 40,91 & 1,39 \\
\hline 17 & $\begin{array}{l}\text { Nieren- und Nebennierentumoren - } \\
\text { Fortgeschrittene und metastasierte } \\
\text { Tumoren }\end{array}$ & 7 & 0 & 6 & 6 & 0 & 1 & 85,71 & 0,93 \\
\hline 19 & Urothelkarzinom - Experimentell & 18 & 0 & 15 & 15 & 1 & 2 & 83,33 & 2,31 \\
\hline 20 & Urothelkarzinom - Diagnostik & 8 & 0 & 5 & 5 & 3 & 0 & 62,50 & 0,77 \\
\hline 21 & $\begin{array}{l}\text { Urothelkarzinom - Prognose und Prä- } \\
\text { diktion }\end{array}$ & 23 & 0 & 10 & 10 & 13 & 0 & 43,48 & 1,54 \\
\hline 22 & $\begin{array}{l}\text { Urothelkarzinom - Therapie nicht-inva- } \\
\text { siver Tumoren }\end{array}$ & 4 & 0 & 3 & 3 & 1 & 0 & 75,00 & 0,46 \\
\hline 23 & $\begin{array}{l}\text { Urothelkarzinom - Therapie invasiver } \\
\text { Tumoren }\end{array}$ & 20 & 0 & 10 & 10 & 10 & 0 & 50,00 & 1,54 \\
\hline 24 & $\begin{array}{l}\text { Urothelkarzinom - Fortgeschrittene } \\
\text { und metastasierte Tumoren }\end{array}$ & 8 & 0 & 8 & 8 & 0 & 0 & 100,00 & 1,23 \\
\hline 26 & Prostatakarzinom - Experimentell & 24 & 0 & 18 & 18 & 5 & 1 & 75,00 & 2,78 \\
\hline 27 & Prostatakarzinom - Diagnostik & 62 & 0 & 27 & 27 & 35 & 0 & 43,55 & 4,17 \\
\hline 28 & $\begin{array}{l}\text { Prostatakarzinom - Prognose und Prä- } \\
\text { diktion }\end{array}$ & 17 & 0 & 10 & 10 & 7 & 0 & 58,82 & 1,54 \\
\hline 29 & $\begin{array}{l}\text { Prostatakarzinom - Therapie lokal be- } \\
\text { grenzter Tumoren }\end{array}$ & 39 & 0 & 18 & 18 & 20 & 1 & 46,15 & 2,78 \\
\hline 30 & $\begin{array}{l}\text { Prostatakarzinom - Therapie lokal fort- } \\
\text { geschrittener Tumoren }\end{array}$ & 3 & 0 & 1 & 1 & 2 & 0 & 33,33 & 0,15 \\
\hline 31 & $\begin{array}{l}\text { Prostatakarzinom - Fortgeschrittene } \\
\text { und metastasierte Tumoren }\end{array}$ & 35 & 0 & 18 & 18 & 17 & 0 & 51,43 & 2,78 \\
\hline 33 & $\begin{array}{l}\text { Tumoren des äußeren Genitale - Ho- } \\
\text { dentumoren }\end{array}$ & 15 & 0 & 9 & 9 & 6 & 0 & 60,00 & 1,39 \\
\hline 34 & $\begin{array}{l}\text { Tumoren des äußeren Genitale - Pe- } \\
\text { niskarziniom }\end{array}$ & 9 & 0 & 9 & 9 & 0 & 0 & 100,00 & 1,39 \\
\hline 36 & Andrologie - Sexuelle Dysfunktion & 15 & 0 & 8 & 8 & 7 & 0 & 53,33 & 1,23 \\
\hline 37 & $\begin{array}{l}\text { Andrologie - Männergesundheit/ } \\
\text { Hypogonadismus }\end{array}$ & 9 & 0 & 3 & 3 & 6 & 0 & 33,33 & 0,46 \\
\hline 38 & Andrologie - Infertilität & 9 & 0 & 6 & 6 & 3 & 0 & 66,67 & 0,93 \\
\hline 39 & Kinderurologie & 16 & 2 & 9 & 11 & 5 & 0 & 68,75 & 1,70 \\
\hline
\end{tabular}




\section{Einführung zum Thema}

Tab. 1 Wissenschaftliche Beiträge zum DGU-Kongress 2016 (Fortsetzung)

Themen- Themen

ID

(1)

\begin{tabular}{ll}
\hline $40 \quad \begin{array}{l}\text { Urologie der Frau/ } \\
\text { Beckenbodenerkrankungen }\end{array}$ \\
\hline 42
\end{tabular}

\begin{tabular}{|llllll|}
\hline 42 & Infektionen/Entzündungen & 17 & 0 & 9 & 9 \\
\hline 44 & Urolithiasis - Experimentell & 5 & 1 & 4 & 5
\end{tabular}

$45 \quad$ Urolithiasis - Diagnostik

$46 \quad$ Urolithiasis - Therapie

$47 \quad$ Uro-Imaging

$49 \quad$ Operationstechniken-Offene

50 Operationstechniken-Endoskopische

Techniken

\begin{tabular}{|llllllllll}
\hline 51 & Operationstechniken - Laparoskopie & 2 & 0 & 1 & 1 & 1 & 0 & 50,00 & 0,15 \\
\hline 52 & Operationstechniken - Roboterchirur- & 13 & 6 & 4 & 10 & 3 & 0 & 76,92 & 1,54
\end{tabular}

gie

\begin{tabular}{|c|c|c|c|c|c|c|c|c|c|}
\hline 53 & Urologische Rehabilitation & 2 & 0 & 0 & 0 & 2 & 0 & 0,00 & 0,00 \\
\hline 54 & Palliativmedizin/Supportive Therapie & 7 & 0 & 3 & 3 & 4 & 0 & 42,86 & 0,46 \\
\hline 55 & Prävention, Umwelt- und Komplemen- & 3 & 0 & 0 & 0 & 3 & 0 & 0,00 & 0,00 \\
\hline
\end{tabular}

55 Prävention, Umwelt- und Komplementärmedizin

\begin{tabular}{|c|c|c|c|c|c|c|c|c|c|}
\hline 56 & Soziologie und Psychosomatik & 6 & 0 & 1 & 1 & 5 & 0 & 16,67 & 0,15 \\
\hline 57 & $\begin{array}{l}\text { Krankenhausökonomie/DRG/ Qualitäts- } \\
\text { sicherung }\end{array}$ & 7 & 0 & 4 & 4 & 3 & 0 & 57,14 & 0,62 \\
\hline 58 & Interessante Fälle („Albträume“) & 16 & 0 & 10 & 10 & 6 & 0 & 62,50 & 1,54 \\
\hline 59 & Geschichte der Urologie & 8 & 0 & 8 & 8 & 0 & 0 & 100,00 & 1,23 \\
\hline & Gesamt & 648 & 12 & 354 & 366 & 273 & 9 & 56,48 & 1,20 \\
\hline
\end{tabular}

Anzah

einge-

Video Oral Ange-

nommen

Abge-

lehnt

gene/zusam-

mengefasste

Beiträge

Beiträge

8

$9 \quad 0 \quad 5 \quad 5$

$\begin{array}{llllll}25 & 0 & 19 & 19 & 6\end{array}$

$6 \quad 0 \quad 6 \quad 6$

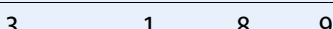

$\begin{array}{lll}1 & 8 & 9 \\ 0 & 3 & 3\end{array}$

0

$\begin{array}{lll}0 & 52,94 & 1,39\end{array}$

0

$100,00 \quad 0,77$

$55,56 \quad 0,77$

$76,00 \quad 2,93$

$100,00 \quad 0,93$

$69,23 \quad 1,39$

